\title{
Sphere-to-rod Transition of Block Copolymer in Solution Induced by Variation of Concentration
}

Xiaoli Zhan, Bi Chen, Kang Chung, Qinghua Zhang, Yiwen Chen, Qian

Wu, Pei Wang*

Key Laboratory of Advanced Textile Materials and Manufacturing Technology of Ministry of Education, Zhejiang Sci-Tech University, Hangzhou 310018, China

\begin{abstract}
A short-chain triblock copolymer $\mathrm{EO}_{9}-\mathrm{DMS}_{7}-\mathrm{EO}_{9}$ was synthesized by coupling reaction of allyl-terminated poly(ethylene oxide) and Si-H-terminated poly(dimethylsiloxane). The structure and purity of synthesized copolymer was carefully characterized. Self-assembly behavior of EO9-DMS $_{7}-\mathrm{EO}_{9}$ triblock copolymer in water was investigated. And it was found that along with the increase of copolymer concentration, morphology of self-assembled aggregates transits from sphere to rod. A plausible understanding of the morphology transition for the investigated triblock copolymer was proposed.
\end{abstract}


Self-assembly of amphiphilic copolymers has attracted a great deal of research attention in recent years for their applications or potential applications in many areas.1-10 A variety of parameters including copolymer concentration, copolymer composition and solvent properties have already shown their effect on the morphology of aggregates formed from solutions of amphiphilic copolymers. ${ }^{11-14}$ The morphological transition of self-assembled aggregates could be triggered by proper control of the preparation condition.To this aim, an unambiguous understanding of the transition between aggregate morphologies is very important.

Polysiloxane-based copolymers, as we know, exhibit unique properties such as high hydrophobicity and flexibility, optical transparency, biocompatibility, etc. These characteristics make polysiloxanes interesting as blocks for the preparation of amphiphilic copolymers with properties different from those of many other purely organic polymers. A special example of amphiphilic copolymers based on polysiloxane is the so-called "superspreaders" consisting of trisiloxanes and polyethers. ${ }^{15-16}$ The remarkable surface-active properties of the "superspreaders" have received much attention. Copolymers consisting of polysiloxane and polyether, hence, received increasing research interest. ${ }^{17-18}$

In this paper, we would like to report the synthesis and solution self-assembly study of a short-chain $\mathrm{EO}_{9}-\mathrm{DMS}_{7}-\mathrm{EO}_{9}$ triblock copolymer. Morphology transition of the self-assembled aggregates was found and investigated. The mechanism of morphology transition is discussed.

\section{Experimental}

Triblock copolymer $\mathrm{EO}_{9}-\mathrm{DMS}_{7}-\mathrm{EO}_{9}$ was synthesized by coupling reaction of allylterminated poly(ethylene oxide) and Si-H-terminated polydimethylsiloxanes. Speier's catalyst was used and prepared by dissolving $\mathrm{H}_{2} \mathrm{PtCl}_{6} \cdot 6 \mathrm{H}_{2} \mathrm{O}$ (Sinopharm Chemical Reagent Co.; A.R.) in isopropanol to form $0.01 \mathrm{~mol} / \mathrm{L}$ solution. Toluene (Sinopharm Chemical Reagent Co.; 99.5\%) was distilled from sodium. Methanol (Sinopharm Chemical Reagent, 99\%) was used as the precipitator. Allylterminated polyethylene oxide (PEO) was kindly provided by Clariant Corporation and characterized by GPC before use. Si-H-terminated polydimethylsiloxanes were purchased from Aldrich and characterized before use. After the completion of the hydrosilation reaction, solvent was evaporated and the products were purified by precipitation into dry methanol.

TEM observations were carried out on a JEM-1230 instrument operated at an accelerating voltage at $80 \mathrm{kV}$. The high electron density of poly(dimethylsiloxane) (PDMS), as compared with PEO, gives sufficient contrast for TEM observation 
without the need of special staining techniques. Equilibrium surface tension was measured with a CAM200 tensiometer (KSV).

\section{Results and discussion}

Fig. 1 shows the ${ }^{1} \mathrm{H}$ NMR spectrum and GPC trace of the copolymer. The structure is shown to be well-defined and the purity was proven by GPC with no homopolymer residues detected in the GPC trace. Polydispersity index (PDI) of $\mathrm{EO}_{9}-\mathrm{DMS}_{7}-\mathrm{EO}_{9}$ is characterized to be 1.13..

The variation of the surface tension with the copolymer concentration was determined as shown in Fig. 2. According to Gibbs' law applied to equilibrium systems, we know that the adsorption of the surfactant at the gas/liquid interface leads to a reduction of the surface tension of the solution. From Fig. 2, the equilibrium surface tension above the critical micelle concentration (CMC) could be found lower than $25 \mathrm{mN} \mathrm{m}^{-1}$ which indicated excellent surface activity of EO9-

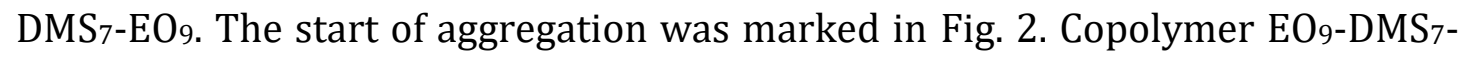
E09 exhibits a CMC value close to $1.5 \mathrm{E}-5 \mathrm{~mol} / \mathrm{L}$.

To induce possible morphology transition, the copolymer concentration of EO9$\mathrm{DMS}_{7}-\mathrm{EO}_{9}$ in water was altered. Fig. 3 shows TEM pictures of aggregates formed in different copolymer concentration varying from 3.37E-4 mol/L $(\sim 22 \mathrm{CMC})$ to $3.37 \mathrm{E}-2 \mathrm{~mol} / \mathrm{L}(\sim 2200 \mathrm{CMC})$. Spherical micelles were observed in relatively low concentration as shown in Fig. 3A. As the concentration increases, oblate elliptical micelles were formed with sparsely distributed spherical micelles as shown in Fig. 3B (white arrows for spherical micelles). Fig. 3C corresponded to aggregates with a concentration of $1.68 \mathrm{E}-2 \mathrm{~mol} / \mathrm{L}$. Intermediate aggregates resulting from collisions of spherical micelle and elliptical ones were observed. And as the concentration increases further to $3.37 \mathrm{E}-2 \mathrm{~mol} / \mathrm{L}$, rod becomes the dominant morphology (Fig. 3D). Color difference could be clearly figured out (may be difficult to observe for black-and-white picture) in each rod-like micelle which may correspond to the difference of electrical density along the rod.

A plausible mechanism of the self-assembly behavior of EO9-DMS7-EO9 was proposed (Scheme 1). Conversion from sphere to oblate ellipse for our investigated copolymer was interpreted as a compromise between the desired interfacial curvature of core-shell-solvent and the conformation of triblock copolymer molecules in aqueous solution. As for triblock copolymer with two hydrophilic blocks and one hydrophobic block, cyclization of the triblock copolymer molecule is anticipated to form spherical micelles. However, the cyclization is obviously unfavorable and it could be easily influenced by lots of parameters that may influence the free energy of the system. It is possible that along with the increase of copolymer concentration, energy balance of the spherical micelles broke and the cyclized triblock copolymers change their conformations, for example to choose a less curved conformation, and hence to 
show a tendency to flatten. As a compromise between the interfacial curvature requirement and the chain conformation requirement, the spherical micelles were transformed into oblate elliptical ones. And with the concentration increasing further, the Brownian motion of nano-size aggregates in solution would lead to the collision of the aggregates with each other. As a result, the oblate elliptical micelles were converted into rod-like aggregates. Intermediate morphologies were detected and the interval dark regions in these rod-like micelles could very possibly be caused by the distribution of copolymers along the rod, the denser the darker. The dark regions, therefore, may correspond to spherical micelles which coexist with oblate elliptical micelles and act as "adhesive" to link oblate elliptical micelles together.

Scheme 1. Mechanism proposed for morphology transition of EO9-DMS7-EO9 in aqueous solution. Core: PDMS. Corona: PEO. Bar:100 nm.

Rod-like aggregates with interval dark regions may not be equilibrium structures. With change of copolymer concentration or other influence parameters, these aggregates could possibly change into uniform rod-like ones. Uniform rod-like aggregates were observed as indicated by white arrow in Fig. 3D.

\section{References}

1. Liu, Y. Q.; Pollock, K. L.; Cavicchi, K. A., Synthesis of poly(trioctylammonium pstyrenesulfonate) homopolymers and block copolymers by raft polymerization. Polymer 2009, 50, 6212-6217.

2. Wang, R.; Peng, J.; Qiu, F.; Yang, Y.; Xie, Z., Simultaneous blue, green, and red emission from diblock copolymer micellar films: A new approach to white-light emission. Chem Commun 2009, 6723-6725.

3. Islam, M. T.; Islam, M. R.; Lim, K. T., A paradigm shift in morphological architecture of peo-b-pfoma semi-fluorinated block copolymer thin films upon facile solvent annealing. Polymer 2011, 52, 5212-5220.

4. Liu, J. H.; Chen, G. S.; Jiang, M., Supramolecular hybrid hydrogels from noncovalently functionalized graphene with block copolymers. Macromolecules 2011, 44, 7682-7691.

5. Wang, R.; Peng, J.; Qiu, F.; Yang, Y., Enhanced white-light emission from multiple fluorophores encapsulated in a single layer of diblock copolymer micelles. Chem Commun 2011, 47, 2787-2789.

6. Brendel, J. C.; Burchardt, H.; Thelakkat, M., Semiconductor amphiphilic block copolymers for hybrid donor-acceptor nanocomposites. J Mater Chem 2012, 22, 
7. Bui, L.; Abbou, S.; Ibarboure, E.; Guidolin, N.; Staedel, C.; Toulme, J. J.; Lecommandoux, S.; Schatz, C., Encapsidation of rna-polyelectrolyte complexes with amphiphilic block copolymers: Toward a new self-assembly route. J Am Chem Soc 2012, 134, 20189-20196.

8. Yin, P. P.; Wu, G.; Dai, R. Y.; Qin, W. L.; Wang, M.; Chen, H. Z., Fine encapsulation of dual-particle electronic ink by incorporating block copolymer for electrophoretic display application. J Colloid Interf Sci 2012, 388, 67-73.

9. Liedel, C.; Lewin, C.; Tsarkova, L.; Boker, A., Reversible switching of block copolymer nanopatterns by orthogonal electric fields. Small 2015, 11, 6058-6064.

10. Doerk, G. S.; Yager, K. G., Rapid ordering in "wet brush" block copolymer/homopolymer ternary blends. Acs Nano 2017, 11, 12326-12336.

11. Jing, R. K.; Wang, G. W.; Huang, J. L., One-pot preparation of aba-type block-graft copolymers via a combination of "click" chemistry with atom transfer nitroxide radical coupling reaction. J Polym Sci Pol Chem 2010, 48, 5430-5438.

12. Han, M.; Kim, H.; Seo, H.; Ma, B. W.; Park, J. W., Photovoltaic efficiency enhancement by the generation of an embedded silica-like passivation layer along the p3ht/pcbm interface using an asymmetric block-copolymer additive. $A d v$ Mater 2012, 24, 6311-6317.

13. Zhao, Y. J.; Pan, D.; Wang, Z., Comparison of four-arm and six-arm star-shaped mpeg-pla block copolymer micelles for drug delivery. J Control Release 2013, 172, E84-E84.

14. Han, B.; Liu, B. Y.; Ding, H. N.; Duan, Z. Y.; Wang, X. H.; Theato, P., Co2-tuned sequential synthesis of stereoblock copolymers comprising a stereoregularityadjustable polyester block and an atactic co2-based polycarbonate block. Macromolecules 2017, 50, 9207-9215.

15. Kang, K.; Kwon, B.; Choi, S. W.; Lee, J.; Kim, D., Properties and morphology study of proton exchange membranes fabricated from the pendant sulfonated poly(arylene ether ketone) copolymers composed of hydrophobic and hydrophilic multi-blocks for fuel cell. Int J Hydrogen Energ 2015, 40, 16443-16456.

16. Vuoriluoto, M.; Orelma, H.; Johansson, L. S.; Zhu, B. L.; Poutanen, M.; Walther, A.; Laine, J.; Rojas, O. J., Effect of molecular architecture of pdmaema-poegma random and block copolymers on their adsorption on regenerated and anionic nanocelluloses and evidence of interfacial water expulsion. J Phys Chem B 2015, 


\section{$119,15275-15286$.}

17. Tran, T. A.; Leonardi, F.; Bourrigaud, S.; Gerard, P.; Derail, C., All acrylic block copolymers based on poly (methyl methacrylate) and poly (butyl acrylate). A link between the physico-chemical properties and the mechanical behaviour on impact tests. Polym Test 2008, 27, 945-950.

18. Xia, Y. D.; Sun, Z. Y.; Shi, T. F.; Chen, J. Z.; An, L. J.; Jia, Y. X., Self-assembly of rodterminally tethered three-armed star-shaped coil block copolymer: Investigation of the presence of the branching in the coil to the self-assembled behavior. Polymer 2008, 49, 5596-5601. 